\title{
Correlation of Biomechanical Features of Keratoconus Patients with Severity of Disease
}

\section{Rana Sorkhabi ${ }^{1}$, Pejman Khatibian, ${ }^{2, *}$}

${ }^{1}$ Professor, Department of Ophthalmology, School of Medicine, Tabriz University of Medical Sciences, Tabriz, Iran

${ }^{2}$ Fellowship Resident, Department of Ophthalmology, School of Medicine, Tabriz University of Medical Sciences, Tabriz, Iran

* Corresponding Author: Pejman Khatibian, Department of Ophthalmology, School of Medicine, Tabriz University of Medical Sciences, Tabriz, Iran. Email: khatibianpejman@gmail.com

\section{Abstract}

Received: 15.09 .2018

Accepted: 17.01.2019

\section{How to Cite this Article:}

Sorkhabi R, Khatibian P.

Correlation of Biomechanical

Features of Keratoconus Patients

with Severity of Disease.

Avicenna J Clin Med. 2019;

25(4): 200-206. DOI: $10.21859 /$

ajcm.25.4.200
Background and Objective: Keratoconus is a disorder in cornea characterized by constant thinning and prominence that can result in vision defect. Due to ultrasonic changes in the corneal structure caused by keratoconus disease, its rigidity and biomechanical properties are subject to some changes. In this regard, the present study was conducted to determine the correlation of biomechanical features of keratoconus patients with the severity of the disease.

Materials and Methods: The case group was selected from the patients with keratoconus who referred to the Corneal Clinic of Nikookari Hospital in Tabriz and the controls had no eye diseases. Corneal biomechanics, corneal hysteresis, and corneal resistance factor were evaluated in all the samples using an ocular response analyzer device.

Results: The present study was carried out on 213 keratoconus eyes and 179 normal eyes. The mean scores of corneal hysteresis were obtained at $13.49 \pm 1.34$ and $7.67 \pm 1.01 \mathrm{mmHg}$ for normal and keratoconus eyes, respectively, which were significantly different between the two groups. The differences between the corneal hysteresis of mild $(8.86 \mathrm{~mm} \mathrm{Hg})$, moderate $(8.30 \mathrm{~mm} \mathrm{Hg})$, and severe $(7.10 \mathrm{~mm} \mathrm{Hg})$ keratoconus eyes were also significant. The mean scores of the corneal resistance factor were reported as $12.68 \pm 1.15$ and $7.36 \pm 1.07 \mathrm{mmHg}$ for normal and keratoconus eyes, respectively, which were significantly different between the two groups. The differences between the corneal resistance factor of mild $(8.55 \mathrm{~mm} \mathrm{Hg}$ ), moderate $(8.05 \mathrm{~mm} \mathrm{Hg})$, and severe $(6.76 \mathrm{~mm} \mathrm{Hg})$ keratoconus eyes were also significant.

Conclusion: The obtained results of the present study showed that corneal hysteresis and corneal resistance factor were lower in keratoconus eyes, compared to those in normal eyes and decreased with the severity of the disease.

Keywords: Corneal Hysteresis, Corneal Resistance Factor, Keratoconus 


\section{ويثگىهاى بيومكانيكى قرنيه در بيماران مبتلا به كراتوكونوس و ارتباط آن با شدت بيمارى}

رعنا سرخابى'، يثزمان خطيبيان r.*

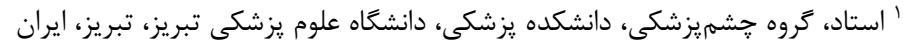

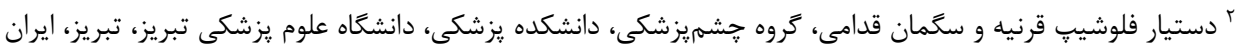

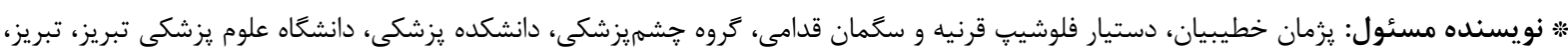
khatibianpejman@gmail.com : ايران. ايميل: جֶيده سابقه و هدف: كراتوكونوس يك اختلال قرنيه است كه با نازكى و برجستهشدن يِيشرونده آن مشخص شده

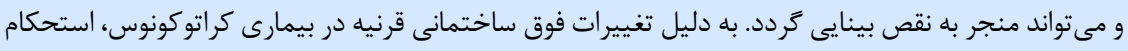

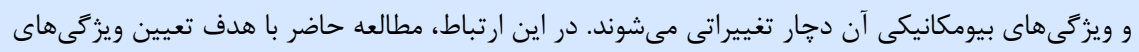

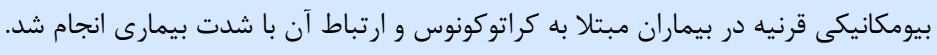

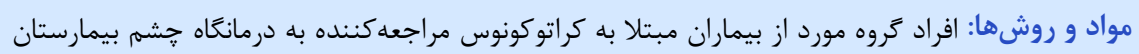

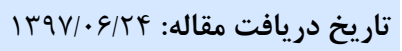

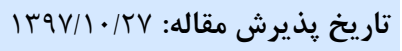
تمامى حقوق نشر براى دانشكاه علوم

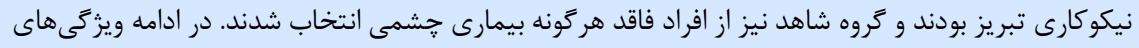

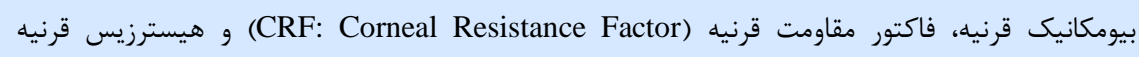

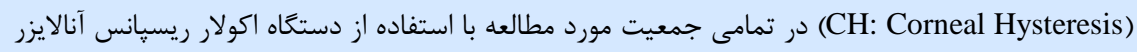
(ORA: Ocular Regression Analyzer)

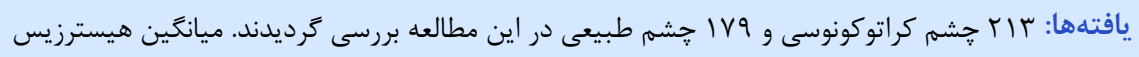

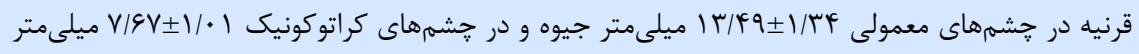

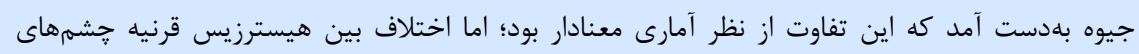

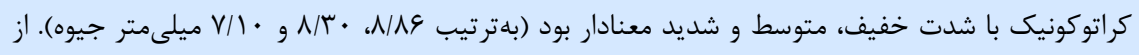

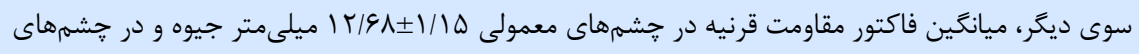

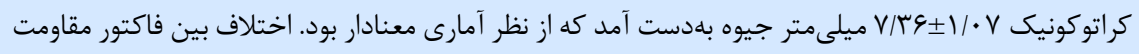

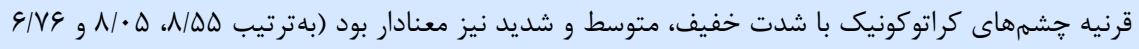
ميلىمتر جيوه). نتيجهَيرى: يافتهها نشان دادند كه هيسترزيس و فاكتور مقاومت قرنيه در جشمهاى كراتوكونوس گإيينتر

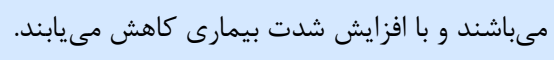
وازًان كليدى: فاكتور مقاومت قرنيه، قوز قرنيه، هيسترزيس قرنيه

يروتئوليتيك كه موجب تخريب كلارن قرنيه مى گردند، مىشود

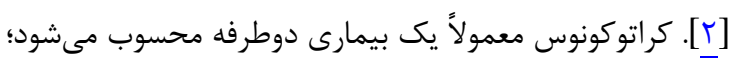

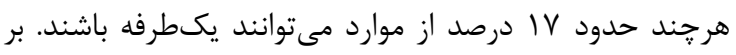

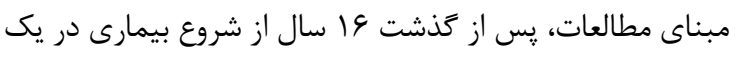

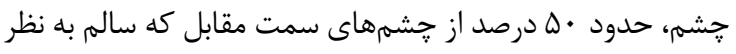

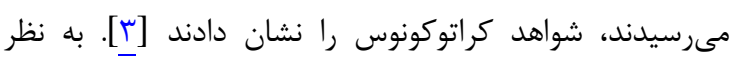
مىرسد كه بافت قرنيه درگير، الاستيسيتى بيشتر و استحكام

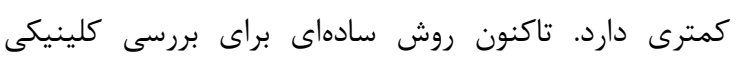

كراتوكونوس يكى از بيمارىهاى جشم است كه با نازك و

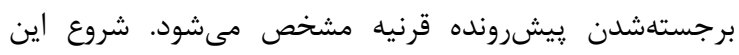

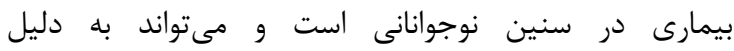

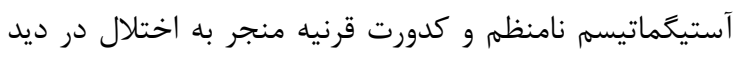

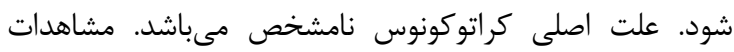

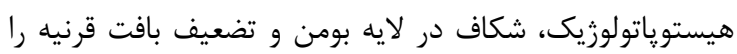

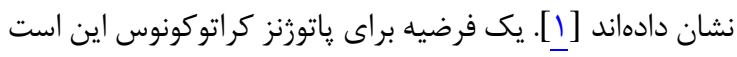

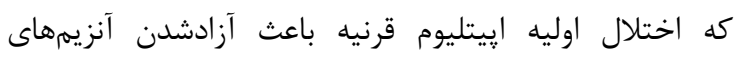


(ORA-G3, Reichert)، هيسترزيس قرنيه و فاكتور مقاومت قرنيه تعيين گرديد. لازم به ذكر است كه اطلاعات بهدستآمده

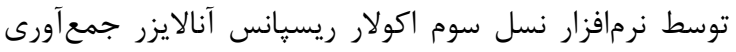

شدند.

شايان توجه مىباشد كه اين مطالعه توسط كميته اخلاق

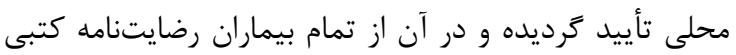

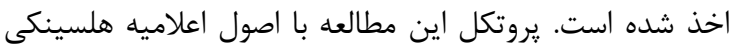
مطابقت دارد. در ادامه بهمنظور تجزيه و تحليل آمارى دادهها از آزمون t و و (Receiver Operating Characteristic) ROC CURVE استفاده گرديد و جندين بسته كامييوترى به كار گرفته

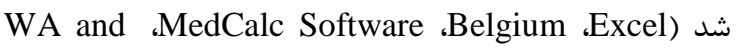
Mariakerke و Microsoft Corp ،Redmond ،MedCalc در اين مطالعه رويكرد آمارى كلى بر مبناى متون استاندارد

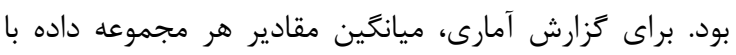

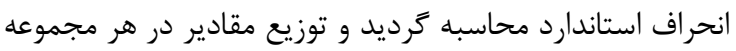

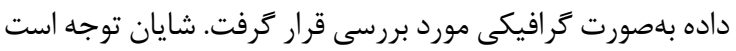

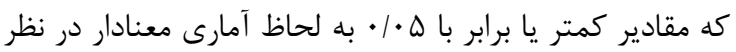

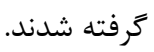

\section{1. إفته}

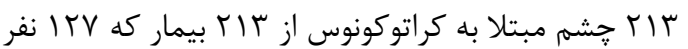

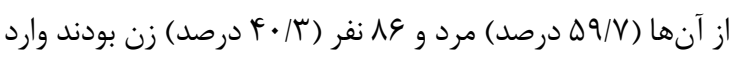

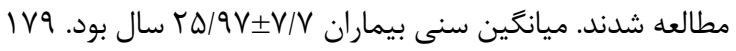

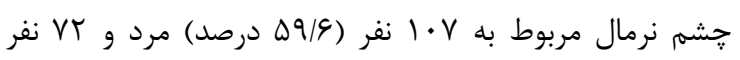

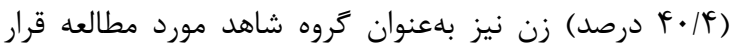

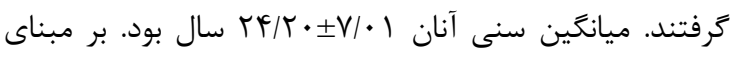

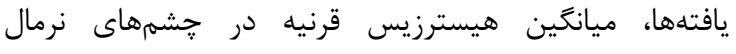
ح جيوه بلهدست آمد كه اين اختلاف از نظر آمارى معنادار بود

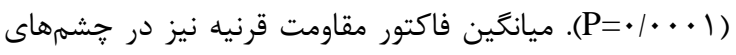

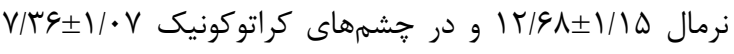

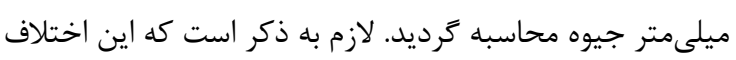

$$
\text { از نظر آمارى معنادار بود (جدول مجيلي (1). }
$$

ويزَّى هاى بيومكانيكى قرنيه ارائه نشده است. اكولار ريسيانس

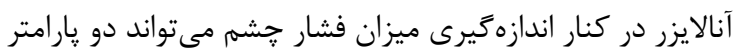

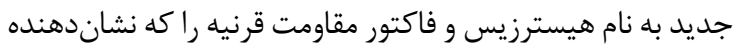

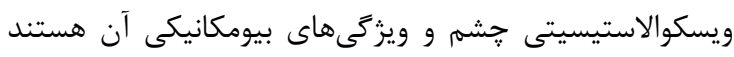

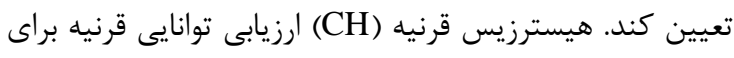
جذب و انهدام انرزى است. فاكتور مقاومت قرنيه (CRF) نيز

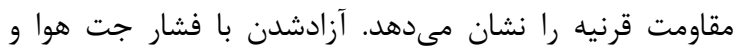
برخورد آن به سطح قدامى محدب قرنيه باعث مسطح و سيس رهن

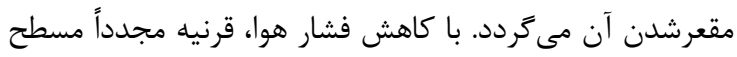

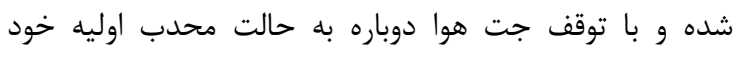

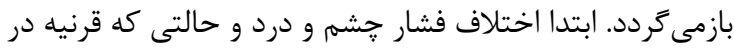

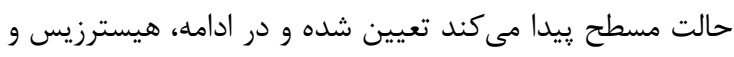

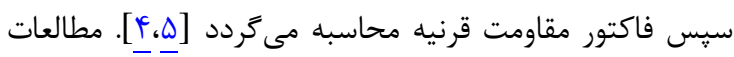

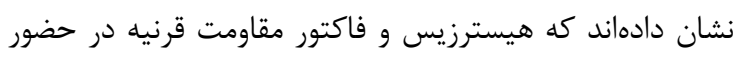

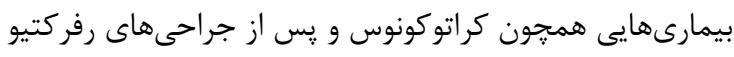

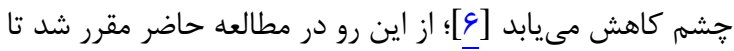

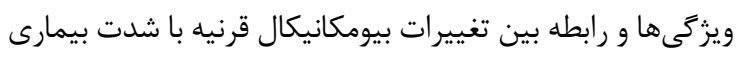

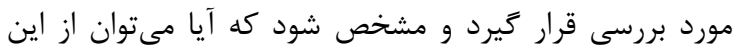

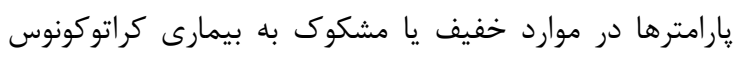

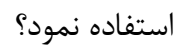

\section{مواد و روشها}

در اين مطالعه مورد- شاهدى، بيماران مبتلا به كراتوكونوس

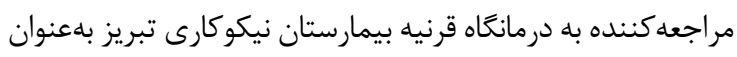

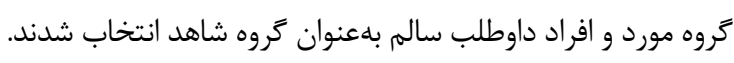
تشخيص كراتوكونوس براساس شرح حال، معاينه و معيارهاى

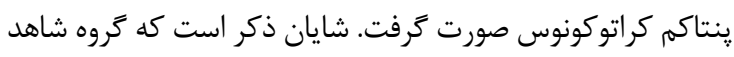

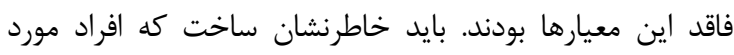

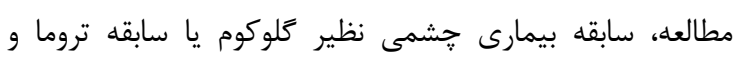

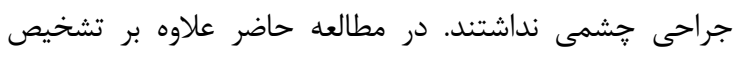
كراتوكونوس تلاش شد تا شدت بيمارى نيز مشخص شود. بيماران

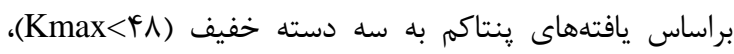

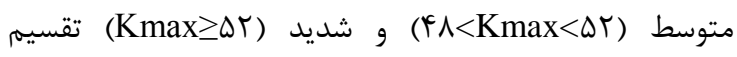
شدند. در ادامه با استفاده از دستعاه اكولار ريسيانس آنالايزر

جدول ا: ويزَّى هاى دموكرافيك و ميانكين متغيرهاى بيومكانيكال قرنيه (ميلىمتر جيوه) در دو گروه مورد مطالعه

\begin{tabular}{|c|c|c|c|}
\hline سطح معنادارى & شاهد & 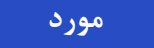 & متغير \\
\hline$<\cdot / \cdot \cdot 1$ & $\wedge \& / \Lambda F$ & qV/DT & Cka (شيب قدامى قرنيه) \\
\hline$<\cdot / \cdot \cdot 1$ & $\mid r / F \Lambda$ & $\mid F / \mu F$ & CKb \\
\hline$<\cdot / \cdot \cdot 1$ & $14 / 4 q$ & $V / 9 V$ & CH \\
\hline$<\cdot / \cdot \cdot 1$ & $\mid r / 9 \Lambda$ & V/RG & CRF C CRتور مقاومت قرنيه) \\
\hline- & $Y F / T \cdot \pm V / \cdot 1$ & $r \omega / q V \pm V / V$ & سن (سال) \\
\hline- & $1 \cdot v$ & I IVV & \\
\hline- & VT & ( & بحسل \\
\hline
\end{tabular}


كراتوكونوس در ابزار Roc Curve نشان داد كه هيسترزيس قرنيه در افتراق بيمارى كراتوكونوس با شدتهاى مختلف داراى دراى

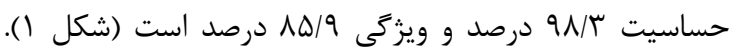
همجنين مشاهده گرديد كه فاكتور مقاومت قرنيه در تشخيص

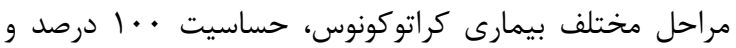

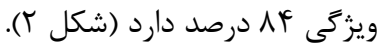

علاوهبراين، اختلاف بين هيسترزيس قرنيه در جشمهاى

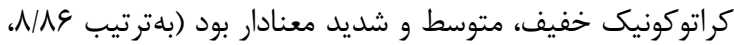

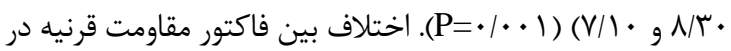
جشمهاى كراتوكونيك خفيف، متوسط و شديد نيز معنادار بهدست

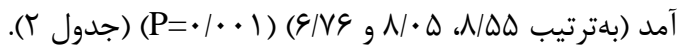

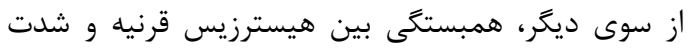

جدول rا: ميانگين متغيرهاى بيومكانيكال قرنيه (ميلىمتر جيوه) در مراحل مختلف بيمارى كراتوكونوس

\begin{tabular}{|c|c|c|c|c|}
\hline سطح معنادارى & شد يد & متوسط & خفيف & متغير \\
\hline$<\cdot / \cdot \cdot 1$ & $1 \cdot r / \Delta 9$ & $91 / \cdot V$ & $\Lambda 9 / \pi \Delta$ & CKa \\
\hline$<\cdot 1 \cdot \cdot 1$ & $-1 \Delta / \pi V$ & $-1 T / 9$. & $-1 Y / 94$ & $\mathbf{C K b}$ \\
\hline$<\cdot / \cdot \cdot 1$ & $V / 1$. & $\Lambda / r$. & $\Lambda / \wedge \&$ & CH \\
\hline$<\cdot / \cdot \cdot 1$ & G/VG & $\Lambda / \cdot \Delta$ & $\Lambda / \Delta \Delta$ & CRF \\
\hline
\end{tabular}

\begin{tabular}{|lc|}
\hline Area under the ROC curve (AUC) & $\cdot / 9 \Delta r$ \\
\hline Standard Error ${ }^{\mathbf{a}}$ & $\cdot / \cdot 1 \cdot 1$ \\
\hline 95\% Confidence interval & \\
z statistic & $\cdot / 9 r \vee-\cdot / 9 \mathrm{Y} /$ \\
\hline Significance level P $($ Area $=\cdot / \Delta)$ & $<r / 1 \cdot 1$ \\
\hline
\end{tabular}

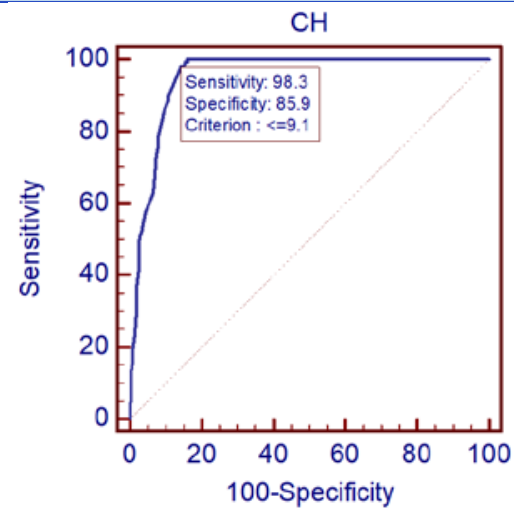

شكل ا: حساسيت و ويزگى هيسترزيس قرنيه در افتراق بيماران دو گروه مورد مطالعه

\begin{tabular}{|c|c|}
\hline Area under the ROC curve (AUC) &.$/ 901$ \\
\hline Standard Error ${ }^{a}$ & $.1 \cdot 114$ \\
\hline 95\% Confidence interval ${ }^{b}$ & $\cdot / 9 r \Delta_{-} \cdot / 9 \gamma \cdot$ \\
\hline z statistic & $r \cdot / r \cdot V$ \\
\hline Significance level $P($ Area $=\bullet / \Delta)$ & $<\cdot|\cdots \cdot|$ \\
\hline
\end{tabular}

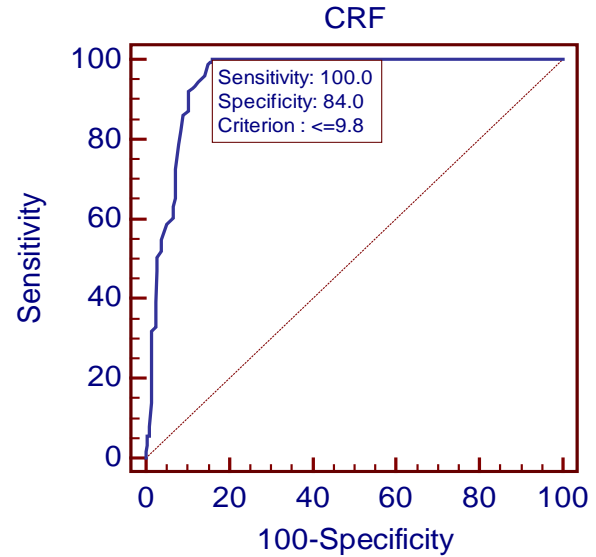

شكل ץ: حساسيت و ويزَگى فاكتور مقاومت قرنيه در افتراق بيماران دو گروه مورد مطالعه 
از هميوشانى زيادى برخوردار مىباشند. اين محققان به اين نتيجه

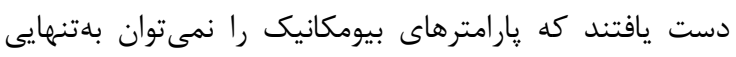

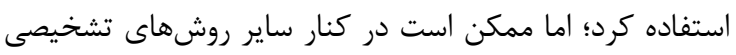

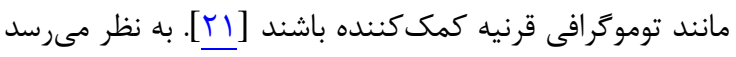
كه فاكتور مقاومت قرنيه (اكر ضخامت قرنيه در نظر كرفته شود)

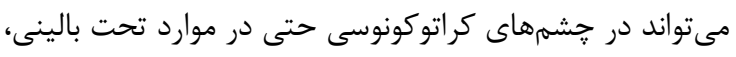

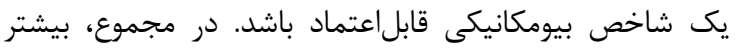

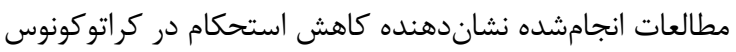

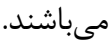

در مطالعه حاضر از اكولار ريسيانس آنالايزر براى آزمايش

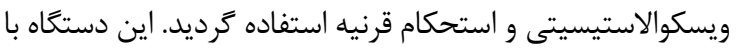

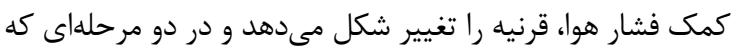

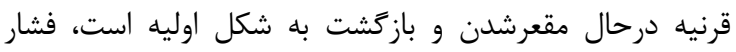

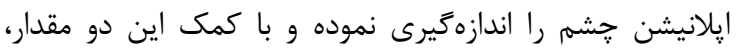

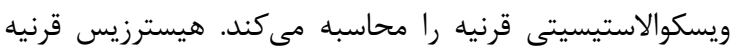

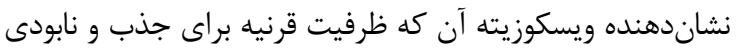
انرزى است، مىباشد. فاكتور مقاومت قرنيه، اندازهيرى اثرات

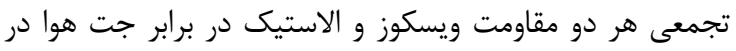

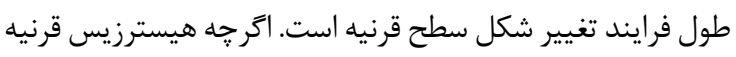

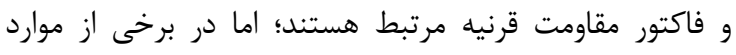

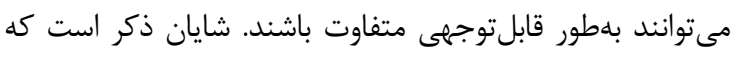

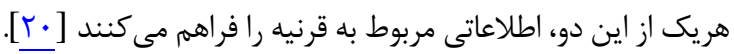
به نظر مىرسد كه ياسخ فورى مربوط به خواص الاستيك كلاثن و ياسخ دير رس مربوط به مشخصات ماتريكس قرنيه باشد. دو فشار

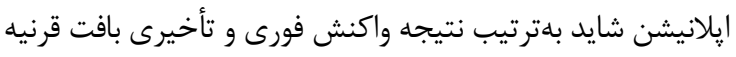

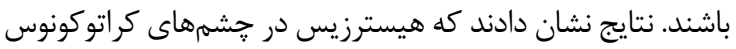

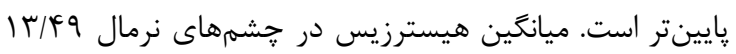

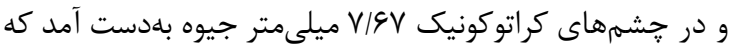

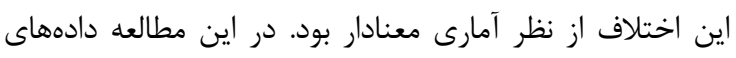

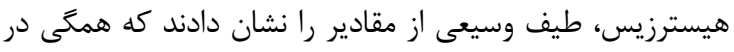

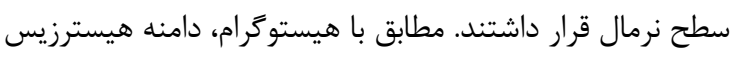

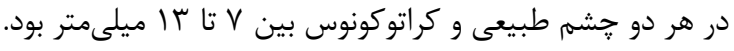

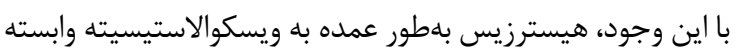

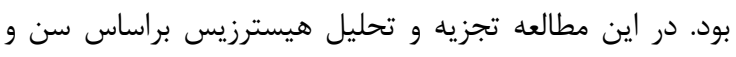

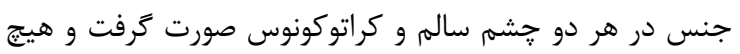

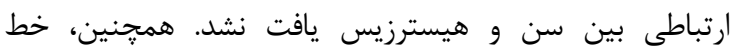

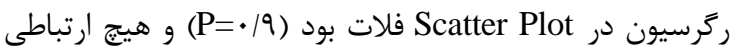
بين هيسترزيس در مردان و زنان مشاهده نشد. تجزيه و وتحليل

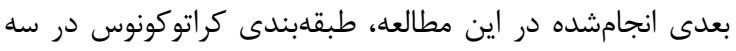

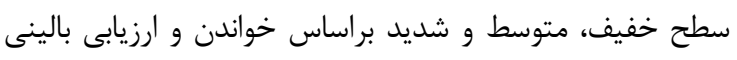

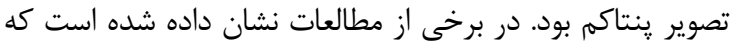

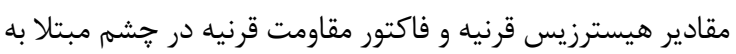

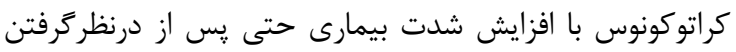

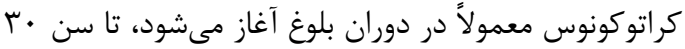

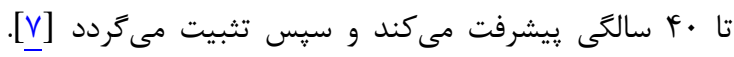

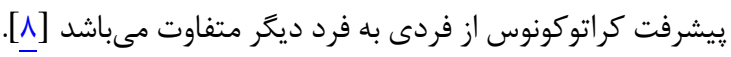

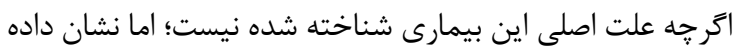

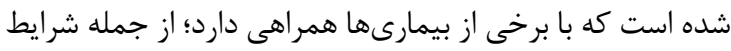

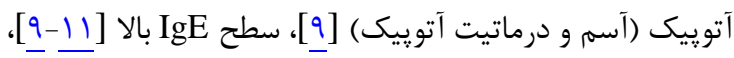
بيمارىهاى غدد درونريز (آديسون و و هييوتيروئيديسم)،

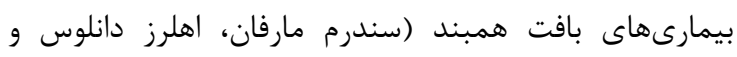

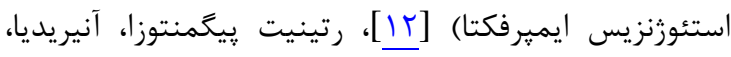
اسكلراى آبى و لبر آموروزيس [بـإئ.

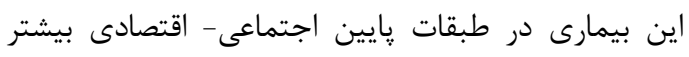

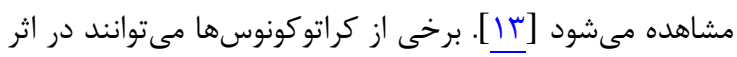

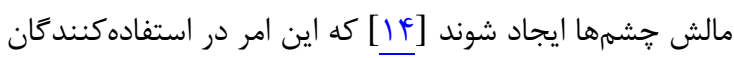

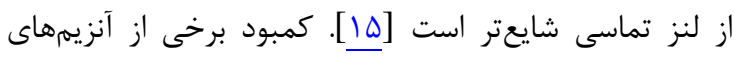

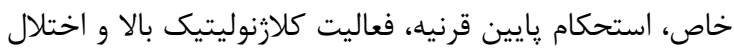

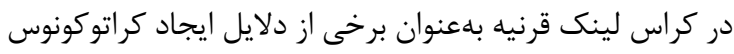

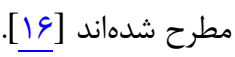
اترجه نازكى استروما در كراتوكونوس با تخريب كلازئ

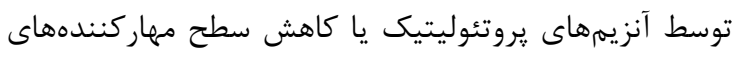

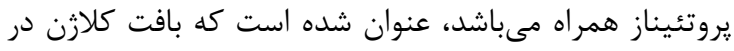

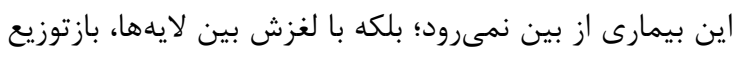
مىشود [IV] دلايل مختلفى براى تغييرات هيستوياتولوزيك قرنيه كزارش شده است؛ از جمله كاهش تعداد فيبرهاى كلازئ نئن

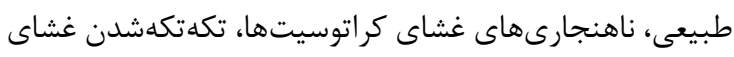

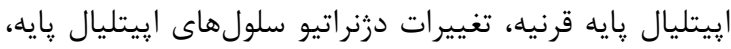
تجزيه لايه بومن و كاهش سطح كلوكوز - צ- فسفات دهيدروزئناز

[11.19]

بسيارى از محققان ت تلاش نمودهاند تأ استحكام

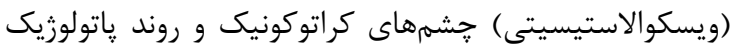

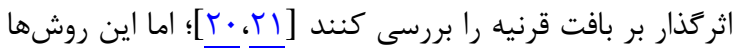

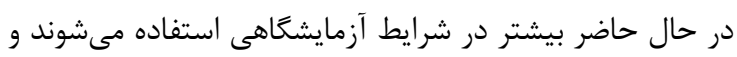

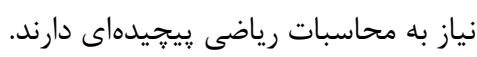

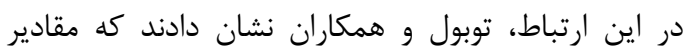

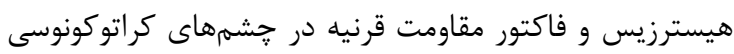

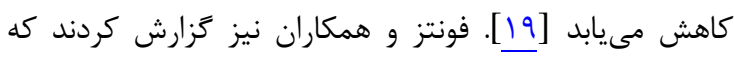

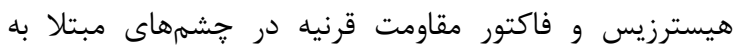

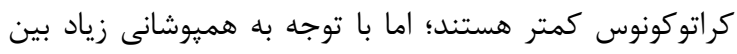

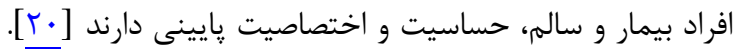

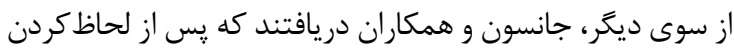

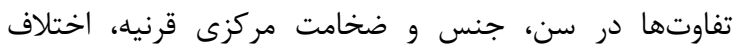
معنادارى در ميانكين هيسترزيس قرنيه و فاكتور مقاومت قرني فرنيه

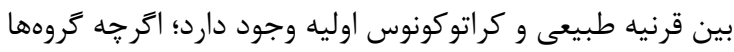


هيسترزيس ممكن است قبل از تغييرات تويوكرافى يا بالينى

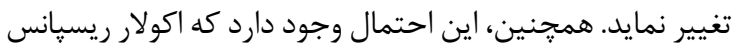

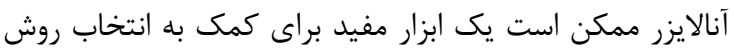
درمان كراتوكونوس باشد.

نتيجه كيرى

هيسترزيس و فاكتور مقاومت قرنيه، مقادير پايينترى را در

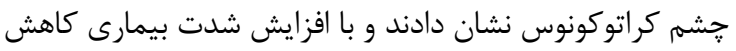

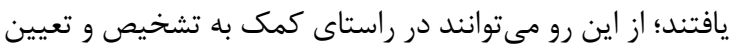

شدت و ييشرفت بيمارى كراتوكونوس مورد استفاده قرار بخيرند.

\section{تشكر و قلروانى}

مقاله حاضر بركرفته از طرح تحقيقاتى مصوب دانشعاه علوم

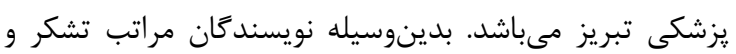

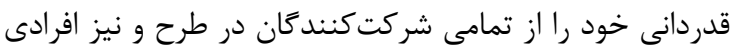
كه در مراحل مختلف انجام يزوهش همكارى داشى داشتند، اعلمام مى دارند. لازم به ذكر مى باشد كه هيج مُونه تضاد منافعى در اين يزوهش كزارش نخرديده است.

\section{REFERENCES}

1. Fournié 1, Touboul D, Arné JL, Colin J, Malecaze F. Keratoconus. J Fr Ophtalmol. 2013;36(7):618-26. PMID: 23911067 DOI: 10.1016/j.jfo.2013.05.004

2. Teng CC. Electron microscope study of the pathology of keratoconus: part 1. Am J Ophthalmol. 1963;55(1):18-47. DOI: 10.1016/0002-9394(63)91645-3

3. Li X, Rabinowitz YS, Rasheed K, Yang H. Longitudinal study of the normal eyes in unilateral keratoconus patients. Ophthalmology. 2004;111(3):440-6. PMID: 15019316 DOI: 10.1016/j.ophtha.2003.06.020

4. Luce DA. Determining in-vivo biomechanical properties of the cornea with an ocular response analyzer. $J$ Cataract Refract Surg. 2005;31(1):156-62. PMID: 15721708 DOI: 10.1016/j.jcrs.2004.10.044

5. Shah S, Laiquzzaman M, Bhojwani R, Mantry S Cunliffe I. Assessment of the biomechanical properties of the cornea with the ocular response analyzer in normal and keratoconic eyes. Invest Ophthalmol Vis Sci. 2007;48(7):3026-31. PMID: 17591868 DOI: $10.1167 /$ iovs.04-0694

6. Saad A, Lteif Y, Azan E, Gatinel D. Biomechanical properties of keratoconus suspect eyes. Invest Ophthalmol Vis Sci. 2010;51(6):2912-6. PMID: 20042662 DOI: 10.1167/iovs.09-4304

7. Zar JH. Biostatistical analysis. $2^{\text {nd }}$ ed. Englewood Cliffs, NJ: Prentice-Hall; 1984.

8. Millodot M, Ortenberg I, Lahav-Yacouel K, Behrman S. Effect of ageing on keratoconic corneas. J Optom. 2016;9(2):72-7. PMID: 26142151 DOI: 10.1016/j.optom. 2015.05.001

9. Davies PD, Lobascher D, Menon JA, Rahi AH, Ruben M. Immunological studies in keratoconus. Trans Ophthalmol Soc U K. 1976;96(1):173-8. PMID: 1070851

10. Kemp EG, Lewis CJ. Immunoglobulin patterns in keratoconus with particular reference to total and specific IgE levels. Br J Ophthalmol. 1982;66(11):717-20. PMID: 7126517

11. Kemp EG, Lewis CJ. Measurement of total and specific IgE levels in the management of a family exhibiting a high incidence of keratoconus. Acta Ophthalmol. 1984;62(4):5249. PMID: 6485750

12. Duke-Elder S, Leigh AG. Diseases of the outer eye. Systems of ophthalmology. Part 2. London: Kimpton; 1964.
CCT: Central (تفاوتها در سن، جنس و ضخامت مركزى قرنيه (Corneal Thickness

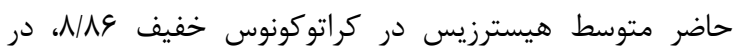

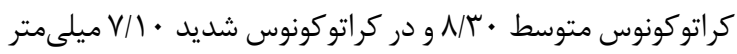

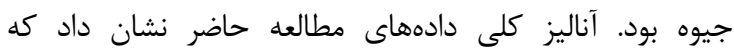

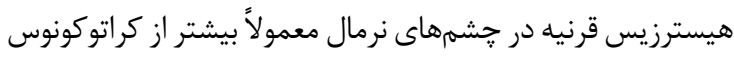

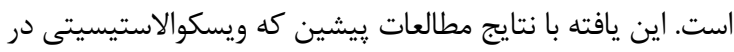
جشمهاى طبيعى و كراتوكونيك را مقايسه كرده بودند، مطابقت داشت [19-rT]

نتايج مطالعه لوس (كه اولين مطالعه كزارششده با استفاده

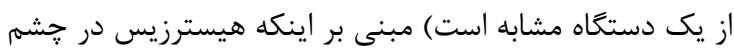

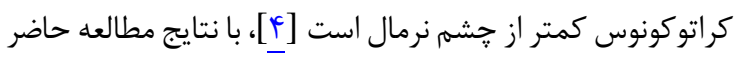

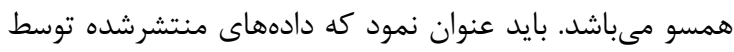

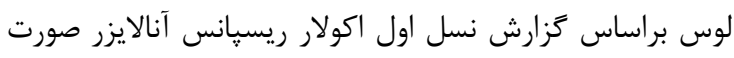

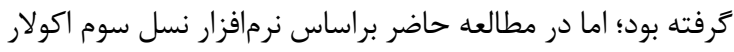

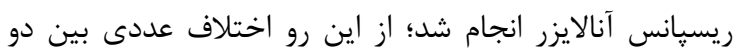

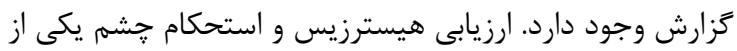

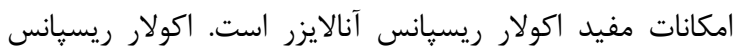
آنالايزر مىتواند براى ارزيابى ييشرفت بيمارى نيز مفيد باشد؛ زيرا

13. Karseras AG, Ruben M. Aetiology of keratoconus. $\mathrm{Br} J$ Ophthalmol. 1976;60(7):523-5. PMID: 952827

14. McMonnies CW. Abnormal rubbing and keratectasia. Eye Contact Lens. 2007;33(6 Pt 1):265-71. PMID: 17993819 DOI: $10.1097 /$ ICL.0b013e31814fb64b

15. Hartstein J. Corneal warping due to wearing of corneal contact lens; a report of 12 cases. Am J Ophthalmol. 1965;60(6):1103-4. PMID: 5857015

16. Krachmer JH, Feder RS, Belin MW. Keratoconus and related noninflammatory corneal thinning disorders. Surv Ophthalmol. 1984;28(4):293-332. DOI: 10.1016/00396257(84)90094-8

17. Davidson AE, S Hayes S, Hardcastle AJ, Tuft SJ. The pathogenesis of keratoconus. Eye (Lond). 2014;28(2):189-95. PMID: 24357835 DOI: 10.1038/eye.2013.278

18. Chi HH, Katzin HM, Teng CC. Histopathology of keratoconus. Am J Ophthalmol. 1956;42(6):847-60. DOI: 10.1016/0002-9394(56)90654-7

19. Touboul D, Bénard A, Mahmoud AM, Gallois A, Colin J, Roberts CJ. Early biomechanical keratoconus pattern measured with an ocular response analyzer: curve analysis. $J$ Cataract Refract Surg. 2011;37(12):2144-50. PMID: 21978610 DOI: 10.1016/j.jcrs.2011.06.029

20. Fontes BM, Ambrósio R Jr, Velarde GC, Nosé W. Ocular response analyzer measurements in keratoconus with normal central corneal thickness compared with matched normal control eyes. J Refract Surg. 2011;27(3):209-15. PMID: 20481414 DOI: 10.3928/1081597X-20100415-02

21. Johnson RD, Nguyen MT, Lee N, Hamilton DR. Corneal biomechanical properties in normal, forme fruste keratoconus, and manifest keratoconus after statistical correction for potentially confounding factors. Cornea. 2011;30(5):516-23. PMID: 21045653 DOI: 10.1097/ICO. 0b013e3181f0579e

22. Galletti JG, Pförtner T, Bonthoux FF. Improved keratoconus detection by ocular response analyzer testing after consideration of corneal thickness as a confounding factor. $J$ Refract Surg. 2012;28(3):202-8. PMID: 22230059 DOI: 10.3928/1081597X-20120103-03

23. Cohen EJ, Myers JS. Keratoconus and normal-tension glaucoma: a study of the possible association with abnormal biomechanical properties as measured by corneal hysteresis. 
Cornea. 2010;29(9):955-70. PMID: 20517150 DOI: 10.1097/ICO.0b013e3181ca363c

24. Piñero DP, Alio JL, Barraquer RI, Michael R, Jiménez R. Corneal biomechanics, refraction, and corneal aberrometry in keratoconus: an integrated study. Invest Ophthalmol Vis Sci. 2010;51(4):1948-55. PMID: 19907021 DOI: 10.1167/iovs.09-4177

25. Vinciguerra R, Ambrósio R Jr, Roberts CJ, Azzolini C, Vinciguerra P. Biomechanical characterization of subclinical keratoconus without topographic or tomographic abnormalities. J Refract Surg. 2017;33(6):399-407. PMID: 28586501 DOI: 10.3928/1081597X-20170213-01 